\title{
Visualizing the Role of Molecular Orbitals in Charge Transport through Individual Diarylethene Isomers
}

\author{
Gaël Reecht, ${ }^{* \dagger}$ Christian Lotze, $^{\dagger}$ Dmytro Sysoiev, ${ }^{\ddagger}$ Thomas Huhn, ${ }^{\ddagger}$ and Katharina J. Franke ${ }^{\dagger}$
}

${ }^{\dagger}$ Institut für Experimentalphysik, Freie Universität Berlin, Arnimallee 14, Berlin 14195, Germany

${ }^{\ddagger}$ Fachbereich Chemie, Universität Konstanz, Universitätsstraße 10, Konstanz 78457, Germany

\author{
Supporting Information
}

ABSTRACT: Diarylethene molecules are prototype molecular switches with their two isomeric forms exhibiting strikingly different conductance, while maintaining similar length. We employed low-temperature scanning tunneling microscopy (STM) to resolve the energy and the spatial extend of the molecular orbitals of the open and closed isomers when lying on a $\mathrm{Au}(111)$ surface. We find an intriguing difference in the extension of the respective HOMOs and a peculiar energy splitting of the formerly degenerate LUMO of the open isomer. We then lift the two isomers with the tip of the STM and measure the current

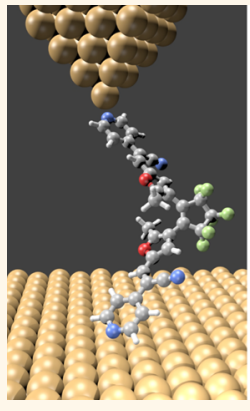

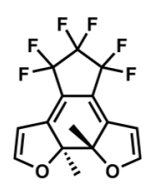
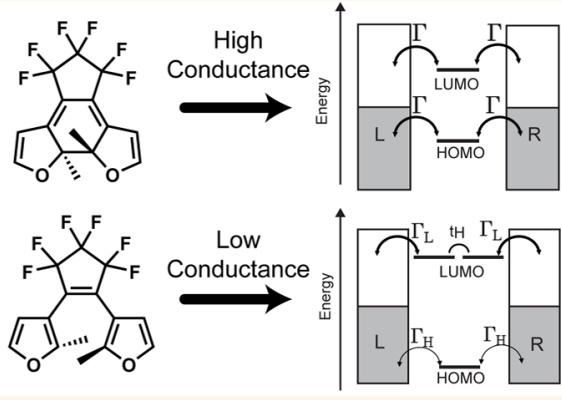
model of the transport, we show that the previously determined orbital characteristics are essential ingredients for the complete understanding of the transport properties. We also succeeded in switching the suspended molecules by the current, while switching the ones which are in direct contact to the surface occurs nonlocally with the help of the electric field of the tip.

KEYWORDS: molecular switch, scanning tunneling microscopy, scanning tunneling spectroscopy, charge transport, diarylethene

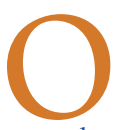

ne challenge for molecular electronics is the creation of single molecule devices whose conductance can be remotely controlled between two distinguishable states. ${ }^{1}$ For this approach, molecular switches are designed with isomeric states, which can be interconverted reversibly by external stimuli, such as, e.g., electrons, ${ }^{2-4}$ photons, ${ }^{5-7}$ or electric fields. ${ }^{8,9}$ For applications in electronic devices, molecular switches must fulfill the following two important requirements: First, the switching behavior needs to be preserved when the molecule is contacted to electrodes. ${ }^{10}$ Second, the conductance of both isomers has to differ drastically, leading to a significant "on-off" ratio.

For this purpose, diarylethenes (DAE) are an interesting molecular class. ${ }^{11}$ DAE can be switched between a ring-closed and a ring-opened form by breaking a $\mathrm{C}-\mathrm{C}$ bond and the conjugation of the $\pi$-electron system. Thereby, the conductance properties are strongly modified, while the geometry of the molecule is only slightly changed, which makes DAE suitable for single molecule devices. Hence, it is not surprising that in the past decade, electron transport through DAE and derivatives was intensively studied. ${ }^{7,10,12-17}$ Depending on the chemical composition of the switching unit, the anchoring groups, or the nature of the electrodes, the switching and conductance properties vary significantly. In particular, it was shown that modifying the electronic structure of the molecule or the electronic coupling to the electrodes can have a drastic effect on the ability of the molecular junction to switch and, likewise, on the "on-off" ratio of the molecular conductance. In order to design the "ideal" molecular switch, it is therefore of crucial importance to have a thorough understanding of the parameters governing the transport properties of the leadmolecule-lead junction.

Measuring the charge transport through individual molecules is a challenging task. It has been well mastered in break junction experiments, where individual molecules are trapped between metallic leads and the junction conductance is measured at variable electrode distance and bias voltage. ${ }^{15,18-20} \mathrm{~A}$ scanning tunneling microscope offers the capability to contact molecules and lift them from the surface, thus resembling traditional break junction experiments, but with a precisely controlled contact point on the molecule. ${ }^{21-24}$ Moreover, the molecules can be characterized when lying on the surface. This yields

Received: September 28, 2016

Accepted: October 24, 2016

Published: October 24, 2016 
complementary information on the molecular structure and molecular orbitals, which is particularly useful for the investigation of molecular switches, as it allows to distinguish the isomers individually. $3,4,8,9,25-27$

Here we investigate 1,2-bis (2-methyl-5-( $Z$-)-(2-cyano-2(pyridin-4-yl)vinyl))furan-3-yl) hexafluorocyclopentene (C5F$4 \mathrm{Py})^{28}$ (Figure 1a) on a $\mathrm{Au}(111)$ surface with low-temperature

a)

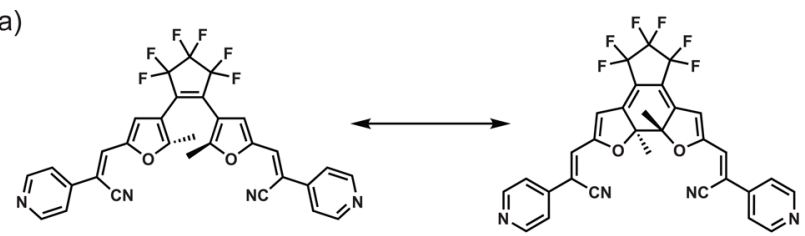

Open Form
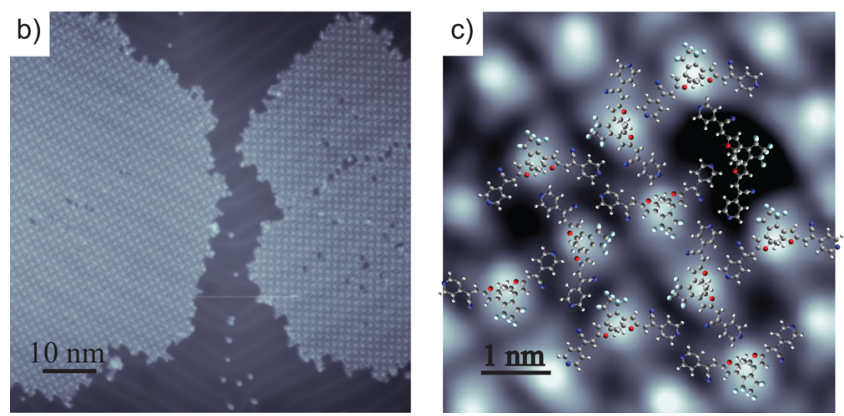

Figure 1. (a) Chemical structure of the open (left) and closed (right) forms of the C5F-4Py molecule. (b) Large-scale STM image $\left(75 \times 75 \mathrm{~nm}^{2}\right)$ of the $\mathrm{Au}(111)$ surface with molecular islands of C5F-4Py $(V=-0.8 \mathrm{~V}, I=40 \mathrm{pA})$. (c) High-resolution STM image $\left(6.5 \times 6.5 \mathrm{~nm}^{2}\right)$ of a molecular island $(V=0.1 \mathrm{~V}, I=40 \mathrm{pA})$. Proposed model of the C5F-4Py calculated in gas phase is added as overlay.

scanning tunneling microscopy (STM), scanning tunneling spectroscopy (STS), and STM-based break junction experiments. C5F-4Py is a DAE with oxygen containing furyl, instead of the commonly used sulfur bearing thiophenyl aryl-rings. The idea behind this choice is to reduce possible interactions with a metallic substrate and, therefore, to facilitate manipulation with the STM tip. First, we show that the molecules exclusively adsorb in the open form, but can be switched into the closed form by voltage pulses from the STM tip. Tunneling spectroscopy and mapping of the spatial orbital distribution allows for an unambiguous identification of the open and closed isomers. We then use the STM tip to controllably lift single molecules from the surface and study their charge-transport properties. As already reported from break-junction experiments with the same molecule, ${ }^{15}$ we observe a large difference in conductance between the open and closed isomers. We model the observed transport properties with the orbitals characteristics, obtained from the STS mapping of the corresponding molecules. In particular, we identify the importance of the localization versus delocalization of transport states and their level splitting, which require hopping between different states along the molecule. Finally, we show that we can induce a switching back into the open form, while the molecule is suspended between the metallic electrodes, by increasing the bias close to the energy of the LUMO state.

\section{RESULTS AND DISCUSSION}

Organization of Molecular Island and Switching. After evaporation of the open C5F-4Py molecules from the chemically pure powder onto a clean $\mathrm{Au}(111)$ surface (see Experimental Methods), we observe ordered molecular islands with a square pattern (Figure $1 \mathrm{~b}$ ). The two islands that we discern on this large-scale STM topography are rotated by about $120^{\circ}$ with respect to each other, in agreement with the 3fold symmetry of the substrate. On the high-resolution image of a molecular island shown in Figure 1c, one molecule has been removed with the STM tip. This allows for a better perception of the molecular arrangement. We propose that one unit cell consists of four molecules, each rotated by $90^{\circ}$ (indicated by the superimposed molecular structure in Figure 1c). The brightest part ("head" or top part) can be associated with the switching unit of the molecule, while the two "legs" with the pyridine groups are lower. This appearance indicates an almost flat-lying configuration due to van der Waals interactions (see Supporting Information). We note an asymmetry at the fluorinated part of the molecule, which is not observed in the gas-phase optimized molecular geometry. In rare cases, single molecules are found on the surface, which show a more symmetric shape (see Supporting Information). This suggests a modification of the molecular geometry within the island, induced by molecule-molecule interactions in the closedpacked structure.

In the STM images shown in Figure $1 b, c$, we observe only one configuration of the molecule but yet can not determine if this is the open or closed isomer (see below). A second one is obtained by applying a voltage pulse in proximity of the molecular islands. Figure 2a,b displays the change in the STM

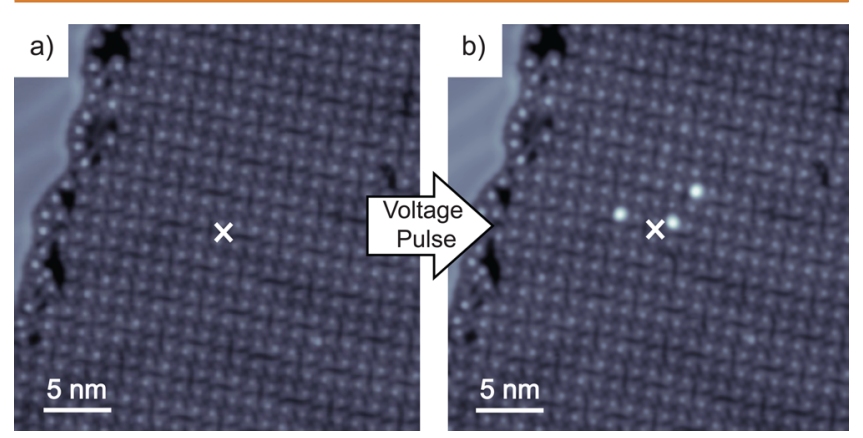

Figure 2. STM images (a) before and (b) after a voltage pulse realized at the position marked by the white cross, with a positive voltage of $10 \mathrm{~V}$ applied for $100 \mathrm{~s}$ and a tip retraction of $5 \mathrm{~nm}$ from the set-point position (STM topographies: $V=0.8 \mathrm{~V}, I=40 \mathrm{pA}$.).

topography before and after a voltage pulse of $10 \mathrm{~V}$ for $100 \mathrm{~s}$, with the tip retracted by $5 \mathrm{~nm}$, has been applied at the position marked by the white cross. After the pulse, some molecules appear brighter with an increased apparent height of $\sim 1 \AA$. These changes can also be observed for smaller voltage pulses when the tip is closer to the surface. But below $7 \mathrm{~V}$, the required tip-surface is so small that a high tunneling current traverses the molecules. In many of these cases, this leads to a destruction of the molecules. Furthermore, we note that switching does occur not only locally underneath the tip but also within a radius of up to $60 \mathrm{~nm}$. The long-range effect suggests the involvement of the electric field in the switching mechanism. ${ }^{8}$ Note that all modified molecules present a similar electronic structure, as will be discussed in the next section. 


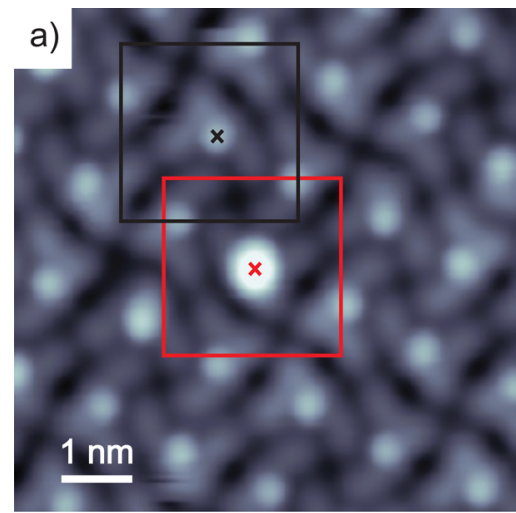

b)

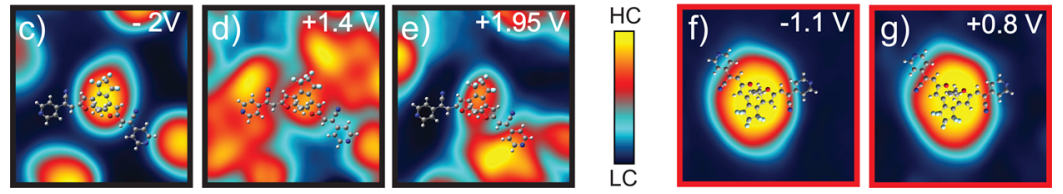

Figure 3. (a) STM image of a molecular island with one modified molecule $\left(7 \times 7 \mathrm{~nm}^{2}, V=0.02 \mathrm{~V}, I=75 \mathrm{pA}\right)$. (b) $\mathrm{dI} / \mathrm{d} V$ spectra recorded on a modified (red) and a nonmodified (black) molecule where indicated with crosses in the STM image. (c-g) Constant current $\mathrm{d} I / \mathrm{d} V$ maps of the area framed by the black $(\mathrm{c}-\mathrm{e})$ and red $(\mathrm{f}-\mathrm{g})$ rectangles in the image in $(\mathrm{a})$. The voltages used for these maps correspond to the energy of the molecular states observed in the $\mathrm{d} I / \mathrm{d} V$ spectra shown in $(b)$. The molecular model is overlaid in these maps for a better perception.

Moreover, back-switching events were sometimes observed, even though we could not find a reliable protocol to induce this process (see Supporting Information). These observations prove that the switched molecules are intact and not destroyed.

Isomer Identification. Until now, we have shown that we can switch the molecule on the surface to a second form. However, no association has been made with the open and closed isomer of the diarylethene C5F-4Py. As the open form is used for the evaporation, it seems likely that molecules assembled on the surface are initially found in their open form (Figure 2a). Hence, the brighter molecule obtained after the voltage pulse might be of the closed form. In order to verify this assumption, we investigate the electronic structure of the two distinct isomers with STS experiments. Figure $3 b$ shows $\mathrm{d} I / \mathrm{d} V$ spectra recorded on top of a molecule before (black) and after (red) switching (see associated crosses in Figure 3a). For the molecule before switching, we observe a resonance at $-2 \mathrm{~V}$, which we assign to tunneling through the highest occupied molecular orbital (HOMO). At positive bias, there is a first increase of conductance around $1.2-1.4 \mathrm{~V}$ and a second one at $2 \mathrm{~V}$ (for discussion of their origin see below). For the molecule after switching, the spectrum is drastically different: In both bias polarities, the molecular orbitals are resonant at lower voltages with $-1.1 \mathrm{~V}$ for the HOMO and $+0.8 \mathrm{~V}$ for the LUMO. Therefore, the HOMO-LUMO gap is significantly smaller after the switching $(1.9 \mathrm{~V})$ than before $(3.2-3.4 \mathrm{~V})$. This difference matches with the switching from a nonconjugated (larger gap) to fully conjugated (smaller gap) molecules and is in agreement with DFT calculations in gas phase (see Supporting Information). It confirms our assumption that molecules before and after switching correspond to open and closed forms, respectively. In the following, open and closed forms will be used to address each form of the molecule.

The energy of the molecular states is not the only difference between the electronic structures of conjugated and nonconjugated molecules. The spatial extension of the molecular orbitals is also expected to be different. Therefore, we record conductance maps of the two isomers at the energies corresponding to their molecular states. The closed form
(Figure $3 \mathrm{f}-\mathrm{g}$ ) exhibits a high conductance signal over almost the whole molecule (except at the extremities of the legs) for both states $(-1.1 \mathrm{~V}$ and $+0.8 \mathrm{~V})$. This agrees with the expectation of delocalized states in conjugated molecules. For the open form, we observe different orbital localizations (Figure $3 \mathrm{c}-\mathrm{e})$. The HOMO (at $-2 \mathrm{~V}$ ) is localized on the switching unit, with almost no conductance on the two legs. The $\mathrm{d} I / \mathrm{d} V$ signal from the two unoccupied states (at +1.4 and $+1.95 \mathrm{~V}$ ) is localized on the switching unit and one leg, but a different leg for each state.

This localization of the molecular states is expected for a nonconjugated molecule. However, we note the surprising asymmetry of the unoccupied states. DFT calculations reveal that the LUMO is doubly degenerate in the gas phase, with the electron density being slightly more localized over one or the other leg (see Supporting Information). ${ }^{29}$ Thus, we suggest that the resonances at +1.4 and $2 \mathrm{~V}$ correspond to these two lifted degenerated molecular orbitals. We ascribe the large splitting between these two states $(0.6 \mathrm{~V})$ to the breaking of symmetry when the molecule is adsorbed on the surface.

Charge-Transport Measurements. By using the atomically precise manipulation capabilities of the STM, we investigate the charge-transport properties of the molecule and its two isomers. Figure 4 explains the principle of this measurement. First, the tip is positioned above one leg of the molecule (white cross on Figure 4a). Then, while the conductance of the junction is recorded, the tip is approached until it contacts the molecule and retracted afterward. Figure $4 \mathrm{c}$ displays the conductance versus tip-sample distance, $z$, for a successful lifting experiment with a closed isomer at a bias voltage of $50 \mathrm{mV}$. Regime I of the curve corresponds to the approach of the tip toward the molecule (sketched in Figure $4 d)$. In this regime, the conductance increases exponentially, while the tip height is reduced, as expected for a tunneling barrier. We identify the contact formation between the tip and the molecule by an abrupt increase of the conductance known as "jump to contact". Subsequently, the tip is retracted, and the conductance decreases, but remains significantly larger than during the approach. This confirms that the molecule is 

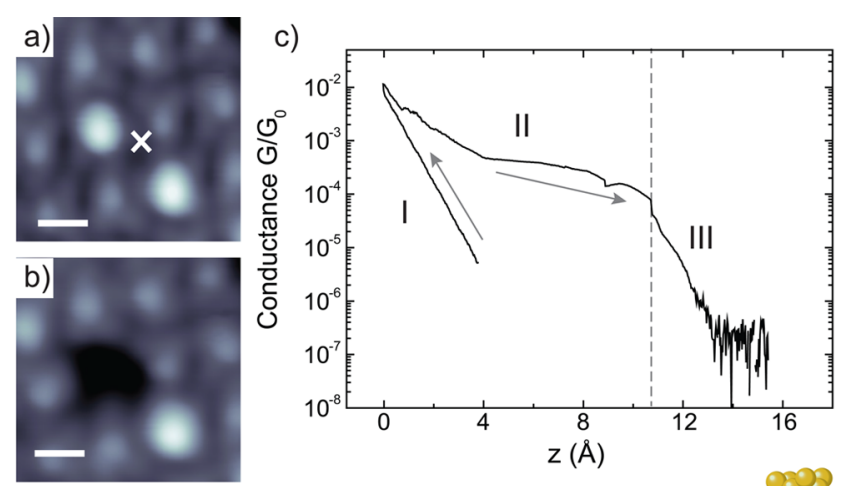

d)
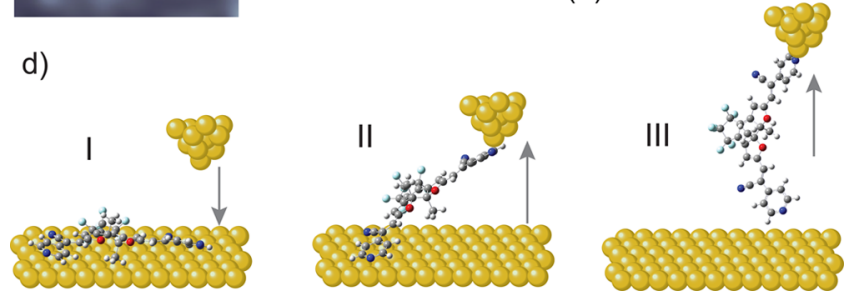

Figure 4. STM images of a molecular island (a) before and (b) after the lifting procedure realized at the white cross location $(7 \times 7$ $\left.\mathrm{nm}^{2}, V=0.1 \mathrm{~V}, I=40 \mathrm{pA}\right)$. Before the image in (b) is recorded, the tip is cleaned to not have the lifted molecule on the tip apex. (c) Evolution of the conductance as a function of $z$, i.e., the tip height, during the lifting of a molecule. This procedure includes an approach of the tip until a contact with the molecule is formed (regime I) and subsequent retraction, until the molecule is detached from the surface (regimes II and III). The bias applied at the junction is $50 \mathrm{mV}$. (d) Scheme of the junction corresponding to the different sections in the curve in (c).

suspended between the tip and the surface (regime II). We note that this regime starts with an exponential decrease. This signifies transport through a tunneling barrier, but with a smaller decay constant than vacuum. Further lifting leads to a quasi-plateau in the conductance. Upon further tip retraction (regime III), we observe a sudden conductance drop, followed by an exponential decrease with a similar slope as for the approach. This behavior indicates that the whole molecule is detached from the surface with an additional gap below the suspended molecule. ${ }^{22,30}$ Scanning the area again after this lifting procedure, indeed, shows that the molecule has been removed from the island (Figure $4 b$ ).
We carried out this lifting procedure for a set of 63 molecules of closed and open states. Figure 5a shows typical conductance traces of closed (red) and open (black) C5F-4Py. As expected, we observe a larger conductance for the closed isomer than for the open isomer. Figure $5 b$ shows a histogram of the conductance, measured on the plateau of the curve (average value in the gray rectangle) for more than 30 molecules of each isomer. The average value obtained for the closed form is $2.8 \times$ $10^{-4} G_{0}$ and $6.1 \times 10^{-6} G_{0}$ for the open form, respectively. The large "on/off" ratio of 45 between the two isomers is in agreement with the results of break junction experiments on the same molecule. ${ }^{15}$

We explain this difference of conductance with a simple analytical model. The most common model used for transport through a molecular junction is the resonant tunneling model as shown in Figure 6a, in which the transport is dominated by one or two molecular levels (HOMO and LUMO in our case). ${ }^{31}$ These levels are defined by two parameters: their energy $\epsilon$ with respect to the Fermi level of the electrodes ( $\mathrm{L}$ and $\mathrm{R}$ ) and $\Gamma$, which describes the strength of the electronic coupling to the metallic electrodes and determines the broadening of the resonant level. For simplicity, we first consider a symmetric coupling to both electrodes, i.e., $\Gamma_{\mathrm{L}}=\Gamma_{\mathrm{R}}$ $=\Gamma=100 \mathrm{meV}$. Thus, the energy-dependent transmission of the junction can be described with a Breit-Wigner distribution for each level:

$$
T(E)=\sum_{i=\mathrm{H}, \mathrm{L}} \frac{4 \Gamma^{2}}{\left[E-\epsilon_{i}\right]^{2}+4 \Gamma^{2}}
$$

Figure 7a displays the $T(E)$ function using this model with the level energies determined from the experimental $\mathrm{d} I / \mathrm{d} V$ spectra, i.e., $-1.1 \mathrm{eV}$ and $+0.8 \mathrm{eV}$ for the closed form (red line) and $-2 \mathrm{eV}$ and $+1.4 \mathrm{eV}$ for the open form (black dashed line). The value of the transmission at the Fermi energy $T\left(E_{\mathrm{F}}\right)$, is only a factor of 3 different for the conductance between the open and closed forms $\left(T_{\mathrm{c}}\left(E_{\mathrm{F}}\right) / T_{\mathrm{o}}\left(E_{\mathrm{F}}\right)\right)$. Hence, it is more than 1 order of magnitude lower than for the experiment. We note that the $\Gamma$ parameter chosen for these curves is $100 \mathrm{meV}$, corresponding to the average broadening of the molecular states for the molecule adsorbed on the surface (Figure $3 \mathrm{~b}$ ). We can expect that the detachment of the molecule from the surface will reduce the broadening and also the value of $\Gamma$. However, reducing the value of $\Gamma$ down to $1 \mathrm{meV}$, a which is already an extreme lower limit for our system, ${ }^{15,20,23,24}$ without
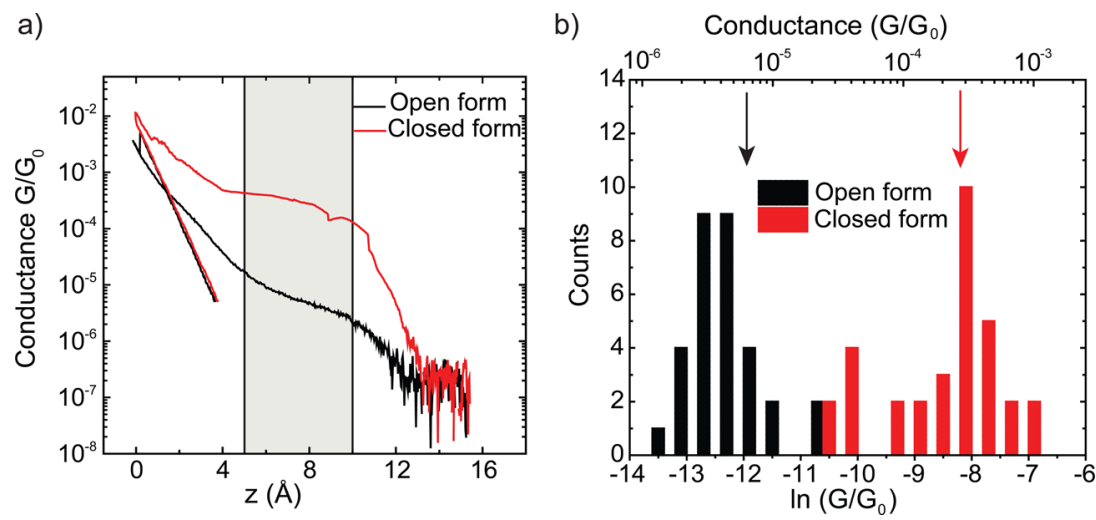

Figure 5. (a) Typical $G(z)$ curves recorded during the lifting of an open (black) and a closed (red) molecule at a bias of $50 \mathrm{mV}$. (b) Histogram of $\ln \left(G / G_{0}\right)$ recorded for 31 (32) open (closed) molecules. The value of the conductance is obtained by averaging the $G(z)$ curve in the plateau region (see gray rectangle in a). The arrows show the position of the average conductance obtained with the statistics. 


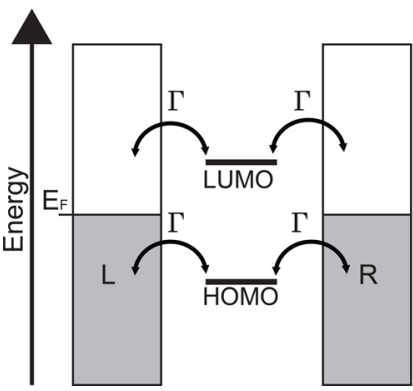

b)

Open Form

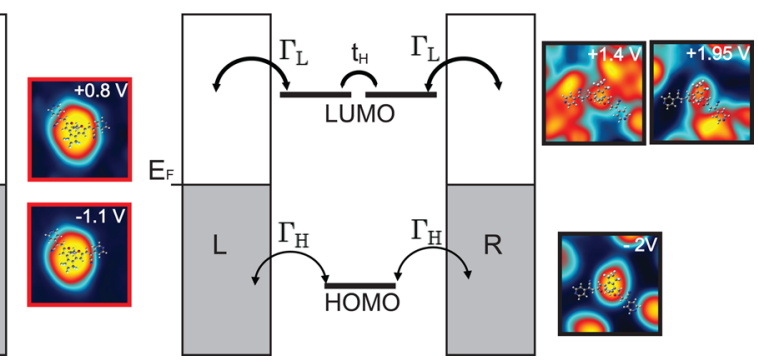

Figure 6. Schemes of the model used to explain differences of conductance. (a) For the closed form, the molecule is represented by two states (HOMO and LUMO), with an electronic coupling $(\Gamma)$, which is the same for both states and both electrodes (L and R). (b) For the open form, the LUMO is represented by two states with a hopping parameter $\left(t_{\mathrm{H}}\right)$, which links them. The electronic coupling to the electrode for the LUMO $\left(\Gamma_{\mathrm{L}}\right)$ is different than the one for the HOMO $\left(\Gamma_{\mathrm{H}}\right)$. The $\mathrm{d} I / \mathrm{d} V$ maps associated with each of the states are shown next to the models.
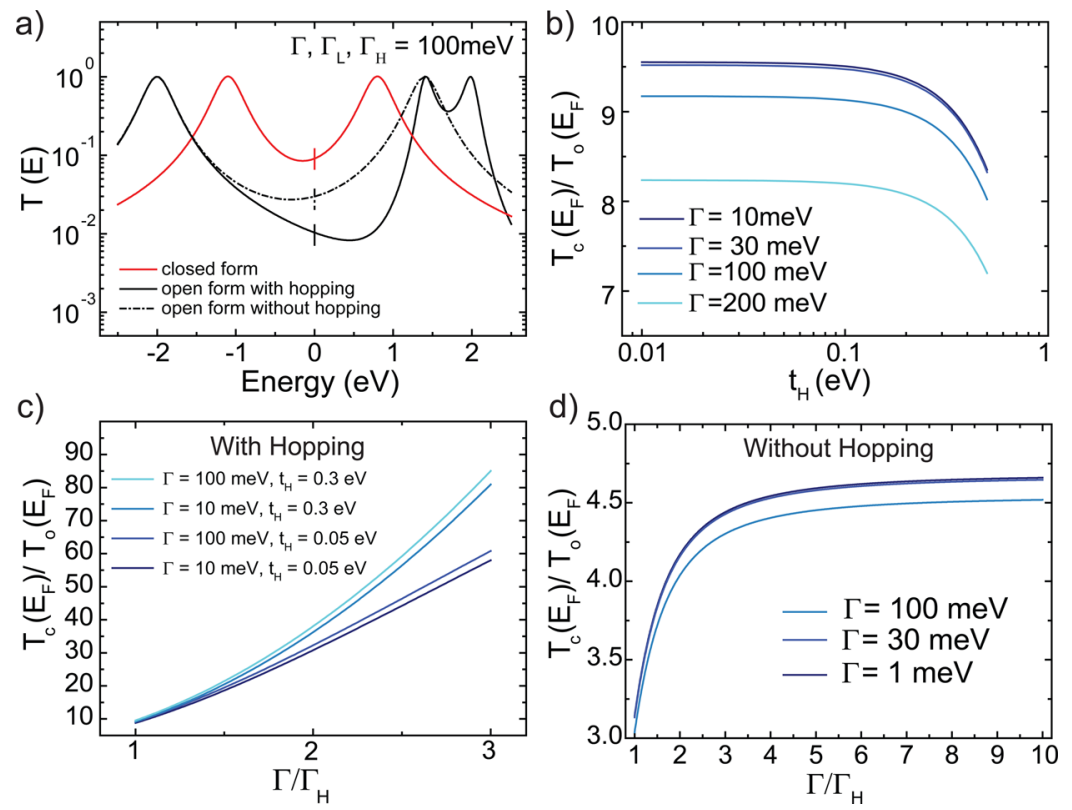

Figure 7. (a) Plot of transmission curves calculated with the model of the simple Breit-Wigner distribution from the states of the closed molecule (red line) and the open one (dashed black line), and with the complete model of the open molecule (full black line). (b) Plot of the ratio of the transmission at the Fermi energy between the open and closed models, as a function of the hopping parameter $t_{\mathrm{H}}$ and for different values of electronic coupling $\left(\Gamma=\Gamma_{\mathrm{L}}=\Gamma_{\mathrm{H}}\right)$. (c) Plot of the ratio of the transmission at the Fermi energy between open and closed models, as a function of the ratio of the electronic coupling $\left(\Gamma / \Gamma_{\mathrm{H}}\right)$, for different values of $t_{\mathrm{H}}$ and $\Gamma$ (with $\left.\Gamma=\Gamma_{\mathrm{L}}\right)$. (d) Plot of the ratio of transmission at the Fermi energy between the open and closed models without considering the hopping model for the open form, as a function of the ratio of the electronic coupling $\left(\Gamma / \Gamma_{H}\right)$, for different values of $\Gamma$ (with $\left.\Gamma=\Gamma_{L}\right)$.

changing the ratio between the open and the closed forms, does not drastically affect the ratio $T_{\mathrm{c}}\left(E_{\mathrm{F}}\right) / T_{\mathrm{o}}\left(E_{\mathrm{F}}\right)$. It only changes between 2.7 and 3.2. Hence, this simple model alone cannot account for the large difference of conductance between the two isomers.

As seen in the experiments above, the different HOMOLUMO gap is not the only difference between the conjugated and nonconjugated molecules. We have already realized above that the double degeneracy of the LUMO of the open isomer was lifted on the surface with each component being localized on a different leg. Naively, the transport thus can be considered by resonant tunneling through coupled molecular orbitals. This is, e.g., similar to the transport through a dihydrogen bridge. ${ }^{32}$ The corresponding model is sketched in Figure $6 \mathrm{~b}$, with the LUMO defined by the two on-site levels coupled together by a hopping parameter $t_{\mathrm{H}}$. Each state is coupled to the nearby electrode by the broadening parameter $\Gamma . t_{\mathrm{H}}$ is associated with the energy splitting of the two states $\Delta E$, such as $\Delta E=2 t_{\mathrm{H}}$. Thereby, the transmission function for the open-form LUMO states is given by ${ }^{32,33}$

$$
T_{\mathrm{L}}(E)=\frac{4 \Gamma^{2} t_{\mathrm{H}}{ }^{2}}{\left[\left(E-\epsilon_{\mathrm{L}}+t_{\mathrm{H}}\right)^{2}+\Gamma^{2}\right]\left[\left(E-\epsilon_{\mathrm{L}}-t_{\mathrm{H}}\right)^{2}+\Gamma^{2}\right]}
$$

where $\epsilon_{\mathrm{L}}$ is the energy of the LUMO without splitting, i.e., $\approx 1.7 \mathrm{eV}$. By adding to this transmission function a usual Breit-Wigner distribution for transport through the HOMO, we obtain the total $T(E)$ curve shown in Figure 7a (black solid line). We observe that in the gap, the transmission is reduced compared to the first model, and the ratio $T_{\mathrm{c}}\left(E_{\mathrm{F}}\right) / T_{\mathrm{o}}\left(E_{\mathrm{F}}\right)$ has increased to $\sim 9$. Note that the hopping parameter for this curve is $t_{\mathrm{H}}=0.3 \mathrm{eV}$, i.e., chosen for the case of the molecule in an 

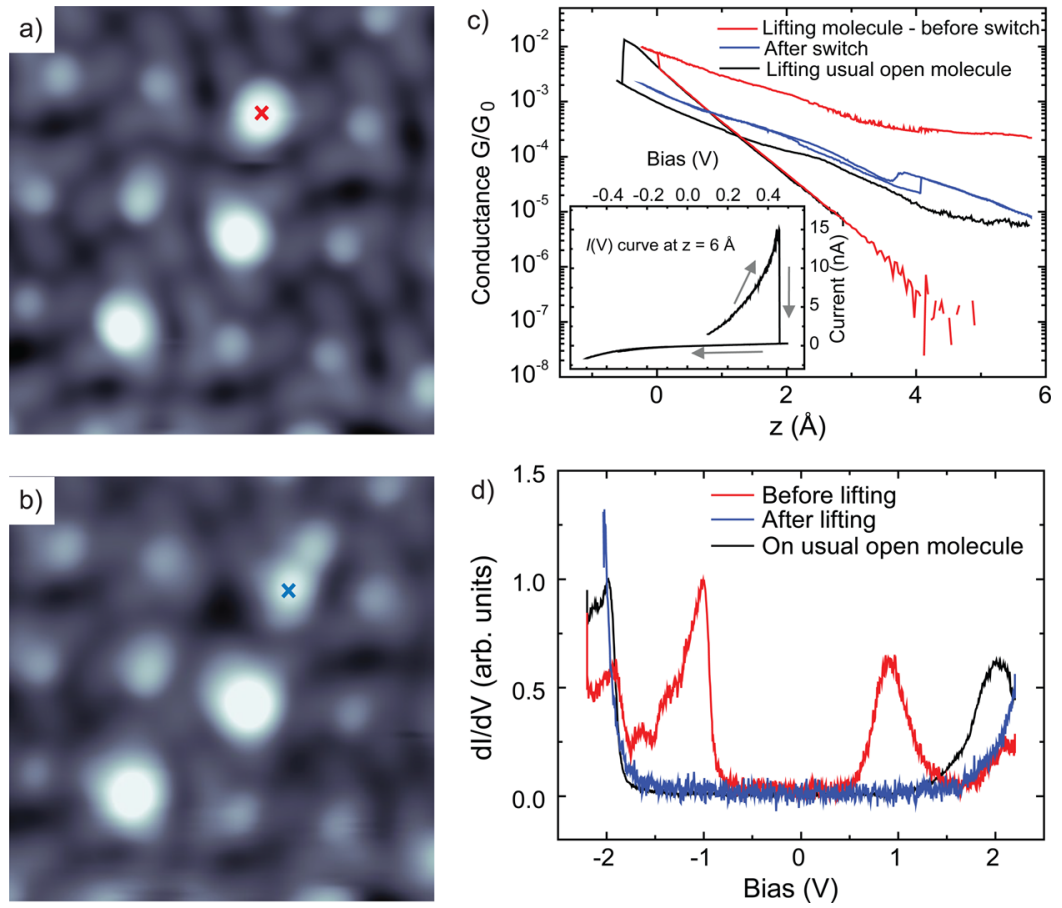

Figure 8. STM images (a) before and (b) after a lifting experiment. (c) $G(z)$ curve at $50 \mathrm{mV}$. The red curve is recorded when a closed molecule (indicated by red cross in a) is lifted. The blue curve is recorded with the same molecule, but after a current drop was measured when a voltage ramp was applied while the molecule was suspended (see inset). The black curve, for comparison, is a typical $G(z)$ curve measured with an open molecule. (d) $\mathrm{d} I / \mathrm{d} V$ spectra recorded on top of the molecule before (red curve) and after (blue curve) the lifting experiment are shown in (c). The black curve, for comparison, is a typical spectrum recorded on an open molecule.

island with a splitting $\Delta E=0.6 \mathrm{eV}$. We may also consider that during the lifting, the molecule is less perturbed by the substrate, and its properties are more similar to those of the free molecule, i.e., a reduced $t_{\mathrm{H}}$. However, changing the value of $t_{\mathrm{H}}$ between 0.5 and $0.05 \mathrm{eV}$ (and also $\Gamma$ ) does not affect the result significantly (see Figure $7 \mathrm{~b}$ ). Finally, considering this hopping model, the calculated ratio $T_{\mathrm{c}}\left(E_{\mathrm{F}}\right) / T_{\mathrm{o}}\left(E_{\mathrm{F}}\right)$ is increased, but still not sufficiently high to explain our experimental results. Next, we also consider the influence of the HOMO state. The HOMO of the open isomer is localized in the center of the molecule (Figure 3c), whereas it is delocalized along the closed isomer. Contacting the molecule at the legs, thus, inevitably leads to a difference in the coupling to the HOMO in the two isomers. ${ }^{34}$ Our picture accounts for this difference by assuming different broadening parameters associated with the HOMOs. Figure $7 \mathrm{c}$ shows the evolution of the ratio $T_{\mathrm{c}}\left(E_{\mathrm{F}}\right) / T_{\mathrm{o}}\left(E_{\mathrm{F}}\right)$ versus the ratio $\Gamma / \Gamma_{\mathrm{H}}$, where $\Gamma_{\mathrm{H}}$ is the broadening parameter for the HOMO state of the open isomer (see Figure $6 \mathrm{~b}$ ). We observe that $T_{\mathrm{c}}\left(E_{\mathrm{F}}\right) / T_{\mathrm{o}}\left(E_{\mathrm{F}}\right)$ increases quickly and reaches the value of the experimentally observed difference of the conductance $(\approx 45)$ for a ratio $\Gamma / \Gamma_{\mathrm{H}}$ between 2 and 3 .

We cannot determine a precise value for the ratio $\Gamma / \Gamma_{\mathrm{H}}$, as we do not know the exact value of $\Gamma$ and $t_{\mathrm{H}}$. Nevertheless, in a realistic range of values for these two parameters, the qualitative description of the system does not change (see different curves in Figure 7c). The disparity of conductance measured experimentally cannot be exclusively explained with a lower coupling for the HOMO state, as shown by Figure $7 \mathrm{~d}$ nor by exclusively taking into account the hopping process for the LUMO state. Instead, both aspects have to be considered.

We now turn back to the conductance $G(z)$ curves in Figure $5 \mathrm{a}$ of the individual molecules. Here, we find indications that our above-described model is indeed correct and involves the spatial characteristics of HOMO and LUMO of the isomers. First, the conductance at the contact $(z=0)$, which mainly depends on the electronic coupling to the electrodes, i.e., $\Gamma$, is lower for the open form, in agreement with the more localized nature of the HOMO in this isomer. On average, we find a factor of 4 of disparity between the open and the closed forms (see Supporting Information). During the lifting, the conductance decreases faster with $z$ for the open form than for the closed one, to reach the ratio of 45 just before the molecules are completely detached from the surface (plateau regime). This dependence of the conductance during the lifting reveals the intrinsic ability of the molecule to transport electrons, which is regulated by the electronic structure of the molecule, energetically and spatially. This is described by the additional hopping parameter, which was included to describe transport through the split LUMO of the open isomer. Thus, our analytical model depicts also these two different contributions, with the influence of $\Gamma_{\mathrm{H}}$ for the electronic coupling and the difference in the energy of the states and the hopping parameter for the intrinsic properties of the molecule.

Note that quantum interferences could in principle affect the electron transport through the open isomer. ${ }^{35,36}$ However, this would require a considerable overlap term between the HOMO and the LUMO. But considering the large energy difference between the states and their drastic localization, the overlap between the HOMO and LUMO should be strongly reduced, and quantum interferences are unlikely.

Switching Events during Molecular Lifting. While a high on-off conductance ratio is important for a molecular device, another challenging key issue for the application of molecular switches is the ability to change the conductance of the molecule while it is bridging the electrodes. ${ }^{10}$ For this purpose, we lift a closed isomer as shown in Figure 8a, with the 
principle presented previously (Figure 4). But instead of lifting it completely, the tip retraction is stopped around $z=6 \AA$, while the molecule is still suspended between tip and surface (red line in Figure 8c).

At low bias, the junction is stable, and no spontaneous change is observed. But when the bias is ramped up, a huge current drop occurs between 0.4 and $0.6 \mathrm{eV}$ (see inset in Figure $8 \mathrm{c})$. To check the conductance properties after this change, a new $G(z)$ curve is recorded (blue line in Figure 8c), which shows a similar behavior as $G(z)$ curves recorded during the lifting of open molecules (black line). This suggests that the molecule switched from the closed to the open form. In order to confirm this, the molecule is placed back on the surface. It is difficult to draw conclusions about the molecular structure alone from the STM image recorded after this manipulation (Figure $8 b$ ), due to a possibly different adsorption of the molecule. However, from STS measurements, the change of conformation is more convincing (Figure 8d). The spectrum after the switching (blue) resembles the usual spectra recorded on open molecules (black curve) and thus suggests the successful switching back.

This experiment proves that the C5F-4Py molecule can be switched, and i.e., its conductance while it is directly connected to electrodes. The required voltage for this switching event corresponds to the onset of the LUMO state. Switching from the closed to the open state was rarely observed for molecules adsorbed on the surface. In the flat-lying configuration, the switching is probably quenched by substrate-molecule or molecule-molecule interactions, whereas the lifting reduces these interactions. Unfortunately, no switching from the open to closed form was observed when the molecule was suspended between the electrodes. The required voltage corresponding to the molecular orbitals of the open form might be too high to be accessed in the experiments, instead the molecule is detached or destroyed.

\section{CONCLUSION}

We found a direct correlation of electronic structure and transport properties of individual diarylethene molecules (C5F$4 \mathrm{Py}$ ), which can be captured in a simple analytical model. We showed that the as-adsorbed molecules reside in their open form on a $\mathrm{Au}(111)$ surface, but can undergo a ring-closing reaction in the presence of an electric field of the STM tip. The two isomers exhibit distinct differences in the energy and the spatial distribution of the molecular orbitals. Hence, they feature an ideal model system to study transport properties through molecules of different electronic structure but similar geometrical length. The STM allows to choose a precharacterized molecule from the surface and then measure the conductance through this very molecule anchored to the STM tip. Similar to previous experiments, we observe a high ratio of conductance between the closed and the open forms. While extensive DFT calculations are often performed to calculate conductance ratios, we propose a purely analytical model based on the results of our STS experiments. This analytical model takes into account the differences of energy and localization of the molecular transport states as well as the electronic coupling to the electrodes and, thus, explains the observed conductance ratio. It may seem surprising that the molecular orbital properties of the flat-lying molecule are very similar to the lifted one. We ascribe this to the three-dimensional nature and O-terminated switching unit of the diarylethene, which prohibits a very strong hybridization of the molecular states with the substrate. The electronic coupling to the leads (STM tip and substrate) in the lifted configuration does then have a similar impact. Thus, by combining the imaging, spectroscopic, and manipulation abilities of the STM, we succeed in correlating the electronic structure and the transport properties of a molecular switch. Finally, we also showed that the molecule can be switched back to the open form while it is lifted by applying a voltage close to the LUMO energy.

\section{EXPERIMENTAL METHODS}

The C5F-4Py diarylethene was synthesized according to ref 28. The $\mathrm{Au}(111)$ sample was cleaned by repeated $\mathrm{Ne}+$ sputtering and annealing cycles. The molecules were evaporated from a Knudsen cell at $440 \mathrm{~K}$ onto the $\mathrm{Au}(111)$ surface held at room temperature in ultrahigh vacuum. The tungsten STM tip was covered with gold by indentation into the clean surface. The quality of the tip was checked on the clean surface by observing the gold surface state in spectroscopy. All STM measurements were done in a home-built STM at a temperature of $4.8 \mathrm{~K}$. Differential conductance spectra were recorded with an open feedback-loop using lock-in detection with a modulation frequency of $910 \mathrm{~Hz}$ and a root-mean-square modulation amplitude of $10 \mathrm{mV}$. For the lifting of the molecules, the vertical manipulations (approach and retraction) are realized with a speed around $1 \AA /$ s.

\section{ASSOCIATED CONTENT}

S Supporting Information

The Supporting Information is available free of charge on the ACS Publications website at DOI: 10.1021/acsnano.6b06559.

Observation of isolated diarylethene molecules. Spectrum in logarithmic scale for determination of substratemolecule interaction. DFT simulation of the molecule in gas phase. Statistics of the conductance at the point of contact formation. Observation of switching back events (PDF)

\section{AUTHOR INFORMATION}

\section{Corresponding Author}

*E-mail: greecht@zedat.fu-berlin.de.

\section{Notes}

The authors declare no competing financial interest.

\section{ACKNOWLEDGMENTS}

We gratefully acknowledge funding by the German research foundation within the framework of the SFB 658 and the European Research Council for the ERC grant "NanoSpin". We thank Elke Scheer for suggesting to study these molecules.

\section{REFERENCES}

(1) Caroll, R. L.; Gorman, C. B. The Genesis of Molecular Electronics. Angew. Chem., Int. Ed. 2002, 41, 4378-4400.

(2) Cai, L.; Cabassi, M. A.; Yoon, H.; Cabarcos, O. M.; McGuiness, C. L.; Flatt, A. K.; Allara, D. L.; Tour, J. M.; Mayer, T. S. Reversible Bistable Switching in Nanoscale Thiol-Substituted Oligoaniline Molecular Junctions. Nano Lett. 2005, 5, 2365-2372.

(3) Choi, B.; Kahng, S.; Kim, S.; Kim, H.; Kim, H.; Song, Y. J.; Ihm, J.; Kuk, Y. Conformational Molecular Switch of the Azobenzene Molecule: A Scanning Tunneling Microscopy Study. Phys. Rev. Lett. 2006, 96, 156106.

(4) Liljeroth, P.; Repp, J.; Meyer, G. Current-Induced Hydrogen Tautomerization and Conductance Switching of Naphthalocyanine Molecules. Science 2007, 317, 1203-1206. 
(5) Katsonis, N.; Kudernac, T.; Walko, M.; van der Molen, S.; van Wees, B.; Feringa, B. Reversible Conductance Switching of Single Diarylethenes on a Gold Surface. Adv. Mater. 2006, 18, 1397.

(6) Snegir, S. V.; Yu, P.; Maurel, F.; Kapitanchuk, O. L.; Marchenko, A. A.; Lacaze, E. Switching at the Nanoscale: Light- and STM-TipInduced Switch of a Thiolated Diarylethene Self-Assembly on $\mathrm{Au}(111)$. Langmuir 2014, 30, 13556-13563.

(7) Jia, C.; Migliore, A.; Xin, N.; Huang, S.; Wang, J.; Yang, Q.; Wang, S.; Chen, H.; Wang, D.; Feng, B.; Liu, Z.; Zhang, G.; Qu, D.-H.; Tian, H.; Ratner, M. A.; Xu, H. Q.; Nitzan, A.; Guo, X. Covalently Bonded Single-Molecule Junctions with Stable and Reversible Photoswitched Conductivity. Science 2016, 352, 1443-1445.

(8) Alemani, M.; Peters, M.; Hecht, S.; Rieder, K.-H.; Moresco, F.; Grill, L. Electric Field-Induced Isomerization of Azobenzene by STM. J. Am. Chem. Soc. 2006, 128, 14446-14447.

(9) Wirth, J.; Hatter, N.; Drost, R.; Umbach, T. R.; Barja, S.; Zastrow, M.; Rück-Braun, K.; Pascual, J. I.; Saalfrank, P.; Franke, K. J. Diarylethene Molecules on a $\mathrm{Ag}(111)$ Surface: Stability and ElectronInduced Switching. J. Phys. Chem. C 2015, 119, 4874-4883.

(10) van der Molen, S. J.; Liljeroth, P. Charge Transport through Molecular Switches. J. Phys.: Condens. Matter 2010, 22, 133001.

(11) Irie, M. Diarylethenes for Memories and Switches. Chem. Rev. 2000, 100, 1685-1716.

(12) Dulić, D.; van der Molen, S. J.; Kudernac, T.; Jonkman, H. T.; de Jong, J. J. D.; Bowden, T. N.; van Esch, J.; Feringa, B. L.; van Wees, B. J. One-Way Optoelectronic Switching of Photochromic Molecules on Gold. Phys. Rev. Lett. 2003, 91, 207402.

(13) Kudernac, T.; van der Molen, S.; van Wees, B.; Feringa, B. Uniand Bi-Directional Light-Induced Switching of Diarylethenes on Gold Nanoparticles. Chem. Commun. 2006, 36, 3597-3599.

(14) Whalley, A. C.; Steigerwald, M. L.; Guo, X.; Nuckolls, C. Reversible Switching in Molecular Electronic Devices. J. Am. Chem. Soc. 2007, 129, 12590-12591.

(15) Kim, Y.; Hellmuth, T. J.; Sysoiev, D.; Pauly, F.; Pietsch, T.; Wolf, J.; Erbe, A.; Huhn, T.; Groth, U.; Steiner, U. E.; Scheer, E. Charge Transport Characteristics of Diarylethene Photoswitching Single-Molecule Junctions. Nano Lett. 2012, 12, 3736-3742.

(16) Arramel, A.; Pijper, T. C.; Kudernac, T.; Katsonis, N.; van der Maas, M.; Feringa, B.; van Wees, B. J. Reversible Light Induced Conductance Switching of Asymmetric Diarylethenes on Gold: Surface and Electronic Studies. Nanoscale 2013, 5, 9277-9282.

(17) Jia, C.; Wang, J.; Yao, C.; Cao, Y.; Zhong, Y.; Liu, Z.; Liu, Z.; Guo, X. Conductance Switching and Mechanisms in Single-Molecule Junctions. Angew. Chem., Int. Ed. 2013, 52, 8666.

(18) Venkataraman, L.; Klare, J. E.; Nuckolls, C.; Hybertsen, M.; Steigerwald, M. L. Dependence of Single-Molecule Junction Conductance on Molecular Conformation. Nature 2006, 442, 904907.

(19) Quek, S. Y.; Kamenetska, M.; Steigerwald, M.; Choi, H. J.; Louie, S.; Hybertsen, M. S.; Neaton, J. B.; Venkataraman, L. Mechanically Controlled Binary Conductance Switching of a SingleMolecule Junction. Nat. Nanotechnol. 2009, 4, 230.

(20) Zotti, L. A.; Kirchner, T.; Cuevas, J.-C.; Pauly, F.; Huhn, T.; Scheer, E.; Erbe, A. Revealing the Role of Anchoring Groups in the Electrical Conduction Through Single-Molecule Junctions. Small 2010, 6, 1529-1535.

(21) Lafferentz, L.; Ample, F.; Yu, H.; Hecht, S.; Joachim, C.; Grill, L. Conductance of a Single Conjugated Polymer as a Continuous Function of Its Length. Science 2009, 323, 1193.

(22) Fournier, N.; Wagner, C.; Weiss, C.; Temirov, R.; Tautz, F. S. Force-Controlled Lifting of Molecular Wires. Phys. Rev. B: Condens. Matter Mater. Phys. 2011, 84, 035435.

(23) Koch, M.; Ample, F.; Joachim, C.; Grill, L. Voltage-Dependent Conductance of a Single Graphene Nanoribbon. Nat. Nanotechnol. 2012, 7, 713-717.

(24) Reecht, G.; Bulou, H.; Scheurer, F.; Speisser, V.; Mathevet, F.; González, C.; Dappe, Y. J.; Schull, G. Pulling and Stretching a Molecular Wire to Tune its Conductance. J. Phys. Chem. Lett. 2015, 6, 2987-2992.
(25) Comstock, M.; Levy, N.; Kirakosian, A.; Cho, J.; Lauterwasser, F.; Harvey, J. H.; Strubbe, D.; Fréchet, J. J.; Trauner, D.; Louie, S.; Crommie, M. Reversible Photomechanical Switching of Individual Engineered Molecules at a Metallic Surface. Phys. Rev. Lett. 2007, 99, 038301.

(26) Schulze, G.; Franke, K.; Pascual, J. Induction of a Photostationary Ring-Opening Ring-Closing State of Spiropyran Monolayers on the Semimetallic Bi(110) Surface. Phys. Rev. Lett. 2012, 109, 026102 .

(27) Shimizu, T.; Jung, J.; Imada, H.; Kim, Y. Adsorption-Induced Stability Reversal of Photochromic Diarylethene on Metal Surfaces. Chem. Commun. 2013, 49, 8710-8712.

(28) Sysoiev, D.; Fedoseev, A.; Kim, Y.; Exner, T.; Boneberg, J.; Huhn, T.; Leiderer, P.; Scheer, E.; Groth, U.; Steiner, U. Synthesis and Photoswitching Studies of Difurylperfluorocyclopentenes with Extended $\pi$-Systems. Chem. - Eur. J. 2011, 17, 6663.

(29) Zhuang, M.; Ernzerhof, M. Mechanism of a Molecular Electronic Photoswitch. Phys. Rev. B: Condens. Matter Mater. Phys. 2005, 72, 073104.

(30) Haiss, W.; Nichols, R. J.; van Zalinge, H.; Higgins, S. J.; Bethell, D.; Schiffrin, D. J. Measurement of Single Molecule Conductivity Using the Spontaneous Formation of Molecular Wires. Phys. Chem. Chem. Phys. 2004, 6, 4330-4337.

(31) Cuevas, J.; Scheer, E. In Molecular Electronics: An Introduction to Theory and Experiment; World Scientific Publishing Co.: Singapore, 2010.

(32) Cuevas, J. C.; Heurich, J.; Pauly, F.; Wenzel, W.; Schön, G. Theoretical Description of the Electrical Conduction in Atomic and Molecular Junctions. Nanotechnology 2003, 14, R29.

(33) Dalgleish, H.; Kirczenow, G. Interface States, Negative Differential Resistance, and Rectification in Molecular Junctions with Transition-Metal Contacts. Phys. Rev. B: Condens. Matter Mater. Phys. 2006, 73, 245431.

(34) Heurich, J.; Cuevas, J. C.; Wenzel, W.; Schön, G. Electrical Transport through Single-Molecule Junctions: From Molecular Orbitals to Conduction Channels. Phys. Rev. Lett. 2002, 88, 256803.

(35) Markussen, T.; Schiötz, J.; Thygesen, K. S. Electrochemical Control of Quantum Interference in Anthraquinone-Based Molecular Switches. J. Chem. Phys. 2010, 132, 224104.

(36) Guédon, C.; Valkenier, H.; Markussen, T.; Thygesen, K.; Hummelen, J.; van der Molen, S. Observation of Quantum Interference in Molecular Charge Transport. Nat. Nanotechnol. 2012, 7,305 . 